\title{
Correction to: A Standards Organization for Open and FAIR Neuroscience: the International Neuroinformatics Coordinating Facility
}

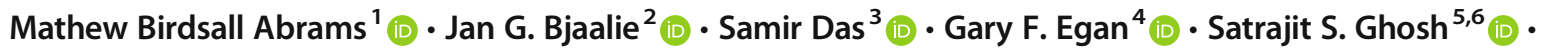 \\ Wojtek J. Goscinski ${ }^{7}$ (D) - Jeffrey S. Grethe ${ }^{8}$ (D) Jeanette Hellgren Kotaleski ${ }^{9}$ (D) Eric Tatt Wei Ho $^{10}$ (D) . $^{\text {. }}$ \\ David N. Kennedy ${ }^{11}$ (D) - Linda J. Lanyon ${ }^{12}$ (D) - Trygve B. Leergaard ${ }^{2}$ (D) - Helen S. Mayberg ${ }^{13}$ (D) Luciano Milanesi $^{14}$ (D) \\ Roman Mouček $^{15}$ (D) - J. B. Poline ${ }^{16}$ (D) Prasun K. Roy ${ }^{17}$ (D) Stephen C. Strother ${ }^{18}$ (D) Tong Boon Tang $^{19}$ (D) \\ Paul Tiesinga $^{20}$ (D) Thomas Wachtler ${ }^{21}$ (D) Daniel K. Wójcik ${ }^{22}$ (D) Maryann E. Martone ${ }^{8}$ (D)
}

Published online: 13 April 2021

(C) The Author(s) 2021

Correction to: Neuroinformatics. https://doi.org/10.1007/s12021-020-09509-0

The original version of this article unfortunately contained mistakes.

(1) These authors were assigned with incorrect affiliation/s: Trygve B. Leergaard, Helen S. Mayberg, Luciano Milanesi, Roman Mouček, J. B. Poline, Prasun K. Roy, Stephen C. Strother, Tong Boon Tang, Paul Tiesinga,
Thomas Wachtler, Daniel K. Wójcik, and Maryann E. Martone.

(2) Serendipitea.Eorld, Hasselby, Sweden should be Serendipitea.World, Hasselby, Sweden.

The original article has been corrected.

Publisher's Note Springer Nature remains neutral with regard to jurisdictional claims in published maps and institutional affiliations.

The online version of the original article can be found at https://doi.org/ 10.1007/s12021-020-09509-0

Mathew Birdsall Abrams

mathew@incf.org

Extended author information available on the last page of the article 


\section{Affiliations}

Mathew Birdsall Abrams ${ }^{1}$ (D) - Jan G. Bjaalie ${ }^{2}$ (D) $\cdot{\text { Samir } \text { Das }^{3} \text { (D) Gary F. Egan }}^{4}$ (D) - Satrajit S. Ghosh ${ }^{5,6}{ }^{(D)} \cdot$ Wojtek J. Goscinski ${ }^{7}$ (D) Jeffrey S. Grethe ${ }^{8}$ (D) Jeanette Hellgren Kotaleski ${ }^{9}$ (D) Eric Tatt Wei $\mathrm{Ho}^{10}$ (D) David N. Kennedy ${ }^{11}$ (D) - Linda J. Lanyon ${ }^{12}$ (D) $\cdot$ Trygve B. Leergaard $^{2}$ (D) $\cdot$ Helen S. Mayberg ${ }^{13}$ (D) $\cdot$ Luciano Milanesi $^{14}$ (D) Roman Mouček ${ }^{15}$ (D) - J. B. Poline ${ }^{16}$ (D) - Prasun K. Roy ${ }^{17}$ (D) - Stephen C. Strother ${ }^{18}$ (ID) Tong Boon Tang ${ }^{19}$ (D) Paul Tiesinga ${ }^{20}$ (D) Thomas Wachtler $^{21}$ (D) $\cdot$ Daniel K. Wójcik $^{22}$ (I) $\cdot$ Maryann E. Martone $^{8}$ (D)

1 INCF Secretariat, Karolinska Institutet, Stockholm, Sweden

2 Institute of Basic Medical Sciences, University of Oslo, Oslo, Norway

3 McGill Centre for Integrative Neuroscience, McGill University, Montreal, QC, Canada

4 Monash Biomedical Imaging, Monash University, Clayton, VIC, Australia

5 McGovern Institute for Brain Research, Massachusetts Institute of Technology, Cambridge, MA, USA

6 Department of Otolaryngology - Head and Neck Surgery, Harvard Medical School Boston, Boston, MA, USA

7 Monash eResearch Centre, Monash University, Melbourne, VIC, Australia

8 Department of Neuroscience, School of Medicine, University of California, San Diego, La Jolla, CA, USA

9 KTH Royal Institute of Technology, School of Electrical Engineering and Computer Science, Stockholm, Sweden

10 Centre for Intelligent Signal and Imaging Research, Institute of Health and Analytics, Universiti Teknologi PETRONAS, Perak, Malaysia

11 Department of Psychiatry, University of Massachusetts Medical School, Worcester, MA, USA

12 Serendipitea.World, Hasselby, Sweden
Nash Family Center for Advanced Circuit Therapeutics, Icahn School of Medicine, New York, NY, USA

14 Institute of Biomedical Technologies, National Research Council (CNR), Milan, Italy

15 Department of Computer Science and Engineering, Faculty of Applied Sciences, University of West Bohemia, Pilsen, Czech Republic

16 Montreal Neurological Institute, Faculty of Medicine and Health Sciences, McGill University, Montreal, Canada

17 Computational Neuroscience \& Neuroimaging Laboratory, School of Bio-Medical Engineering, Indian Institute of Technology (BHU), Varanasi, UP, India

18 Rotman Research Institute, Baycrest Centre, Department of Medical Biophysics, University of Toronto, Toronto, ON, Canada

19 Centre for Intelligent Signal and Imaging Research, Institute of Health and Analytics, Universiti Teknologi PETRONAS, Bandar Seri Iskandar, Malaysia

20 Donders Institute for Brain, Cognition and Behaviour, Radboud University Nijmegen, Nijmegen, Netherlands

21 Department of Biology II, Ludwig-Maximilians-Universität München, Martinsried, Planegg, Germany

22 Laboratory of Neuroinformatics, Nencki Institute of Experimental Biology of Polish Academy of Sciences, Warsaw, Poland 